\title{
Integrated electrical and gas network flexibility assessment in low-carbon multi-energy systems
}

\author{
Stephen Clegg and Pierluigi Mancarella, Senior Member, IEEE
}

\begin{abstract}
In power systems with more and more variable renewable sources, gas generation is playing an increasingly prominent role in providing short-term flexibility to meet netload requirements. The flexibility provided by the gas turbines in turn relies on the flexibility of the gas network. While there are several discussions on the ability of the gas network in providing this operational flexibility, this has not been clearly modelled or quantified. In addition, the gas network may also be responsible for supplying heating technologies, and low-carbon scenarios see a tighter interaction between the electricity, heating and gas sectors, which calls for a holistic multi-energy system assessment. On these premises, this paper presents an original methodology to quantify the flexibility the gas network can provide to the power system, as well as the constraints it may impose on it, with also consideration of different heating scenarios. This is achieved by a novel multi-stage integrated gas and electrical transmission network model, which uses electrical DC OPF and both steadystate and transient gas analyses. A novel metric that makes use of the concept of zonal linepack is also introduced to assess the integrated gas and electrical flexibility, which is then used to impose gas-related inter-network inter-temporal constraints on the electrical OPF. Case studies are performed for the Great Britain transmission system for different renewables and heating scenarios to demonstrate the proposed integrated flexibility assessment methodology, provide insights into the effects of changes to the heating sector on the multi-energy system's combined flexibility requirements and capability, and assess how the electrical network can experience local generation and reserve constraints related to the gas network's lack of flexibility.
\end{abstract}

Index Terms - Power system flexibility, Low-carbon heating, Natural gas network, Optimal power flow, Multi-energy systems, Renewable energy sources.

\begin{tabular}{ll} 
& \multicolumn{1}{c}{ NOMENCLATURE } \\
Index: & Electrical bus \\
$b$ & Conventional non-gas fueled generator \\
$C G$ & Compressor stations \\
$C P$ & Gas turbine \\
$G T$ & Transmission line
\end{tabular}

This paragraph of the first footnote will contain the date on which you submitted your paper for review.

This work was supported by National Grid Gas plc and EPSRC

The authors are with the Electrical Energy and Power Systems Group, School of Electrical and Electronic Engineering, University of Manchester, Manchester, $\quad$ M13 9PL UK stephen.clegg@postgrad.manchester.ac.uk, p.mancarella@manchester.ac.uk)

$\begin{array}{ll}n, n_{i}, n_{j} & \text { Gas network nodes } \\ R G & \text { Renewable generator } \\ z & \text { Linepack zones } \\ \pi & \text { Pipes }\end{array}$

Variables:

A

A

$a, \bar{a}, \underline{a}$

B

$B P_{s}, B P_{e} \quad$ Start/end of gas network balancing period

$c_{C G}, c_{G T}, c_{R G}$ Cost of generation [£/MWh]

$c_{g} \quad$ Cost of natural gas $[£ / \mathrm{MWh}]$

$C_{z} \quad$ Additional gas withdrawal $\left[\mathrm{m}^{3}\right]$

$\overline{C_{z}}, C_{z} \quad$ Upper/lower limit to $C_{z}\left[\mathrm{~m}^{3}\right]$

CGENS $_{b} \quad$ Non-gas conventional generators at bus $b$

$d \quad$ Pipe diameter [m]

$\overline{D_{P}}, \quad$ Max gas demand for generation $\left[\mathrm{m}^{3} / \mathrm{s}\right]$

$\overline{D_{N}}$

$D, D_{n}, D_{\mathcal{N}}$

Max gas demand not for generation $\left[\mathrm{m}^{3} / \mathrm{s}\right]$

Gas demands $\left[\mathrm{m}^{3} / \mathrm{s}\right]$

$D_{n, N}, D_{n, P}$

e

$\overline{E_{Z}}$

$E D_{b}$

$f_{1}, f_{2}$

$f s$

$F$

Non-generation/generation gas demand $\left[\mathrm{m}^{3} / \mathrm{s}\right]$

Speed of sound in gas $[\mathrm{m} / \mathrm{s}]$

Limit to increase in gas generation $[\mathrm{MJ}]$

Electrical demand at bus $b$ [MW]

Objective functions of OPFs

Forecast generator status

Fanning transmission factor

FD Forecast system gas demand $\left[\mathrm{m}^{3} / \mathrm{s}\right]$

$F D_{P} \quad$ Forecast generation gas demand $\left[\mathrm{m}^{3} / \mathrm{s}\right]$

$F D_{N} \quad$ Forecast non-generation gas demand $\left[\mathrm{m}^{3} / \mathrm{s}\right]$

$\mathrm{FH}, \mathrm{FH}_{l} \quad$ Forecast transmission line power flows [MW]

$F L \quad$ Forecast linepack $\left[\mathrm{m}^{3}\right]$

FP, FP Forecast real power output of generators [MW]

$\overline{F P_{R G}} \quad$ Forecast generation capability [MW]

FR $\quad$ Forecast reserve provided by generator [MW]

FS Forecast gas supply $\left[\mathrm{m}^{3}\right]$

FSR Forecast system reserve requirements [MW]

$F S W, F S W_{z}$ Forecast (zonal) linepack swing $\left[\mathrm{m}^{3}\right]$

$F U, F U_{b} \quad$ Forecast net power injection by bus [MW]

GGENS $_{b} \quad$ Gas turbines at bus $b$

$G T_{Z} \quad$ Gas turbines in zone $z$

$H, H_{l} \quad$ Transmission line power flows [MW]

$\overline{H_{l}} \quad$ Transmission line rating [p.u.] 


\begin{tabular}{|c|c|}
\hline$H H V$ & Higher heating value of natural gas $\left[\mathrm{MJ} / \mathrm{m}^{3}\right]$ \\
\hline$J, j$ & Input/target for iterative procedure in Fig. 4 \\
\hline$K_{\pi}$ & Pipe constants $\left[\mathrm{m}^{3 \alpha} \mathrm{s}^{-\alpha} \mathrm{Pa}^{-2}\right]$ \\
\hline$L, L_{z}, L_{\pi}$ & Linepack of system, zone or pipe $\left[\mathrm{m}^{3}\right]$ \\
\hline $\bar{L}, \overline{L_{z}}$ & Upper limit to system (zonal) linepack $\left[\mathrm{m}^{3}\right]$ \\
\hline$\underline{L}, \underline{L_{z}}$ & Lower limit to system (zonal) linepack $\left[\mathrm{m}^{3}\right]$ \\
\hline$M$ & Mass flow rate $[\mathrm{kg} / \mathrm{s}]$ \\
\hline $\mathcal{N}$ & Collection of gas nodes \\
\hline$n_{s}$ & Slack node in gas network analysis \\
\hline$p, p_{n}$ & Pressures in network $[\mathrm{Pa}]$ \\
\hline$p_{\pi, 1}, p_{\pi, 2}$ & Pressures at start and end of pipe $[\mathrm{Pa}]$ \\
\hline$p_{s, 1}, p_{s, 2}$ & Pressures at start and end of pipe section $[\mathrm{Pa}]$ \\
\hline$p_{C P_{\text {in }}}, p_{C P_{\text {ou }}}$ & Pressures at compressor inlet/outlet $[\mathrm{Pa}]$ \\
\hline$\underline{p}, \bar{p}$ & Minimum and maximum system pressures $[\mathrm{Pa}]$ \\
\hline $\bar{p}_{n_{s}}^{*}$ & Pressure at slack node $[\mathrm{Pa}]$ \\
\hline$p_{\mathrm{NTP}}$ & Pressure at normal temperature and pressure $[\mathrm{Pa}]$ \\
\hline $\boldsymbol{P}, P$ & Real power output of generators [MW] \\
\hline$\overline{P_{C G}}, \overline{P_{G T}}$ & Generator capacity $[\mathrm{MW}]$ \\
\hline$\underline{P_{C G}}, \underline{P_{G T}}$ & Minimum stable generation levels [MW] \\
\hline$\overline{\overline{P_{G T_{Z}}}} \overline{-}$ & Upper limit to gas generation increase [MJ] \\
\hline$Q_{\pi}, Q_{C P}$ & Gas flow rate $\left[\mathrm{m}^{3} / \mathrm{s}\right]$ \\
\hline$Q_{s, 1}, Q_{s, 2}$ & Gas flow at ends of pipe section $\left[\mathrm{m}^{3} / \mathrm{s}\right]$ \\
\hline$\overline{R_{C G}}, \overline{R_{G T}}$ & Generator 30 minute ramp capability [MW] \\
\hline$R_{\text {spec }}$ & Specific gas constant $[\mathrm{J} / \mathrm{kg} \mathrm{K}]$ \\
\hline $\mathrm{RGENS}_{b}$ & Renewable generators at bus $b$ \\
\hline$S, S_{n}, S_{\mathcal{N}}$ & Gas supply rate $\left[\mathrm{m}^{3} / \mathrm{s}\right]$ \\
\hline$S R$ & System reserve [MW] \\
\hline$t, t_{0}$ & Time $[\mathrm{s}]$ \\
\hline$T$ & Linepack flexibility utilization period $[\mathrm{s}]$ \\
\hline$T_{D}$ & Time for response in supply flows [s] \\
\hline$\underline{T R}, \underline{T R_{Z}}$ & Lower linepack for transport limits $\left[\mathrm{m}^{3}\right]$ \\
\hline$\overline{\overline{T R}}, \overline{\overline{T R}}_{Z}$ & Upper linepack for transport limits $\left[\mathrm{m}^{3}\right]$ \\
\hline$U, U_{b}$ & Net power injection at each bus [MW] \\
\hline$V$ & Pipe volume $\left[\mathrm{m}^{3}\right]$ \\
\hline$\overline{W_{z}}, \bar{W}$ & Gas and electric flexibility metric $[\mathrm{MJ}]$ \\
\hline $\mathrm{X}$ & Matrix of line impedances [p.u.] \\
\hline$x$ & Distance along gas pipe $[\mathrm{m}]$ \\
\hline$Z, Z_{s}, Z_{s}^{\prime}$ & Compressibility factor of gas in pipe \\
\hline$\alpha$ & Exponent in steady-state flow relation \\
\hline$\beta_{C P}$ & Compression ratio \\
\hline$\Delta T_{E}$ & Time resolution for electrical OPF [s] \\
\hline$\Delta F D_{t_{0}}$ & Change in forecast gas demand [MJ] \\
\hline$\eta_{G T}, \eta_{z}$ & Efficiency of gas turbines \\
\hline $\begin{array}{l}\Theta \\
\Pi_{n}\end{array}$ & $\begin{array}{l}\text { Absolute temperature of gas }[\mathrm{K}] \\
\text { Pipes adjacent to } n\end{array}$ \\
\hline$\rho, \rho_{\mathrm{NTP}}$ & Density of gas $\left[\mathrm{kg} / \mathrm{m}^{3}\right]$ \\
\hline$\tau$ & Time $[\mathrm{s}]$ \\
\hline
\end{tabular}

\section{INTRODUCTION}

$\mathbf{I}^{\mathrm{T}}$ $\mathrm{T}$ is well recognized that with increasing penetration of renewable energy sources such as wind and photovoltaics the need for balancing the power system through flexible resources will increase. In particular, forecast errors and variability in the renewable generation output will require additional reserves and ramping capability to follow net load variation. Furthermore, with various possible scenarios that have been envisaged to carry out decarbonisation of the heating sector (see for instance [1] for the UK), electricity demand will be more and more affected by heating requirements, for example due to electrification of heating via electric heat pumps (EHP) and the introduction of high efficiency combined heat and power (CHP) facilities. In particular, large EHP penetration may lead, amongst others, to even further changes in the net load ramps. It is therefore essential to be able to quantify the power systems flexibility requirements and capability taking into account decarbonisation options not only for the electrical sector, but also for the heating sector.

With regards to options to provide power system flexibility, it is expected that gas turbines will increasingly be the conventional generator of choice to meet variable and unpredicted changes in net demand for balancing purposes [2]. As such, power system flexibility will more and more depend on the gas networks ability to meet the fuel requirements of combined-cycle gas turbines (CCGTs). In particular, rising shares of variable and relatively unpredictable renewables will cause gas turbines to rely increasingly on the gas network's linepack storage should they be required to ramp up at unforeseen times [3]. On the other hand, the changes occurring in the heating sector might also impact substantially on the gas demand and network flexibility requirements and capability in manifold ways, depending on specific technology scenarios. For example, it is likely that electrification of heating will reduce the overall gas demand for direct heating (in gas boilers) and so increase the dominance of power stations' demand in the overall gas flow. Hence, the effects of variable power stations' demands on the gas network will be exacerbated. On the other hand, both EHP and CHP technologies will change the overall volumes and load patterns of gas usage, which will impact on the storage intrinsically available in the pipelines' linepack [4] and therefore on the gas network's ability to provide flexibility and balance supply and demand. The compounded effects that changes in the linepack due to different low carbon heating scenarios might bring about in the presence of large scale penetration of renewables are completely unexplored. This paves the way to the need for developing an integrated electricity and gas network flexibility analysis framework considering future low-carbon electricity and heat options in a truly multi-energy system context [5].

For evaluations of power system flexibility, several papers have been recently written (see for instance [6]-[8]) where flexibility metrics have been developed and the ability of the power system to meet net load requirements assessed, although without consideration for gas network constraints. Impact of changes in the heating sector on the net ramp rates have not been assessed either. Some integrated gas and electrical models [9]-[12] have been proposed that consider constraints between the two networks; in particular, [10] presents examples where gas network limitations impose constraints on electrical generator operation. However, there is no consideration as to gas network limitations on gas turbine ramping and ability to provide system balancing. On the other 
hand, in [12] models have been developed to assess the coordinated daily scheduling of gas turbines and the operation of the gas network, although without accounting for a withinday evaluation of the ability of the gas turbines to change their output or for the flexibility of the gas network to support such ability. In [3] a gas network flexibility model has been developed where an assessment is made to the arbitrage strategies of gas shippers using daily gas balancing; however, there is no consideration for the operational flexibility and the ability of the gas network to respond to changes in supply/demand. In any case, the implications of changes in the heating sector on electrical and/or gas flexibility requirements and capability have never been assessed. In this respect, even previous work of the author on heat-gas-electricity interactions [13] does not assess changes in the heating sector on the net ramp rates or the flexibility implication for the electricity and the gas networks.

The work here aims at bridging the gaps identified above. In particular, it is the first to develop an integrated gas and electric flexibility model, and a relevant flexibility metric, to assess the ability of the transmission networks to react to changes in the power sector due to intermittent renewables including the ability to follow net loads and provide reserve.

The proposed assessment framework and metric evaluate the quantity of gas in the network which is available to gas turbines at their locality and then determines possible limits to the turbine's operation because of a shortfall of gas. In particular, considering the intrinsic storage characteristics available in the gas network's pipelines as a means of evaluating its short-term ${ }^{1}$ flexibility capability, limits are defined in terms of constraints on the additional energy output of the turbine over a period of time. In this respect, as a further key novelty and contribution, specific inter-network intertemporal constraints are formulated and included in classical electrical Optimal Power Flow (OPF) modelling to account for the local flexibility constraints imposed by the gas network, also showing how reduced flexibility in a given area of the network may restrict the ability of gas turbines in that region to provide power system reserve.

The combined flexibility assessment relies on a multi-stage integrated electricity and gas model specifically developed. More specifically, the availability of gas is evaluated using a novel gas network analysis methodology composed of a steady-state gas flow model to assess the network's planned operation and a transient gas flow model for the determination of the continual change to the network's state. On the power system side, gas and electrical transmission network interactions are evaluated using two DC OPFs, namely, the first in the time-ahead scheduling of the gas network operation, and the second in the determination the real-time gas generators' dispatch (with the $2^{\text {nd }} \mathrm{OPF}$ also accounting for the constraints imposed by the gas network and whose results are finally used to determine the actual gas demand).

\footnotetext{
${ }^{1}$ By "short-term" flexibility in this paper we mean the ability to respond to intraday changes in the gas network (in case prompted by the electrical network) over the forthcoming few hours; in these timescales, other sources of flexibility, e.g., gas storage, have limited ability to respond.
}

As mentioned earlier, another novel contribution is assessing the use of alternative heat sources as part of a lowcarbon energy system in terms of impact and flexibility implications on the integrated electricity and gas system, thus leading to a truly multi-energy system assessment. In order to do so, hourly heat profiles at a regional level are used, which points out, in particular, the changes in net load ramps that are required in the presence of electrification of heating.

The paper is structured as follows. Section II describes the main features of the integrated gas and electrical network modelling introduced for the analysis, while Section III discusses the proposed methodology for integrated gas and electrical flexibility assessment and the relevant metric put forward. The system-level modelling of the heating sector for the purposes of integrated heat-gas-electrical multi-energy flexibility studies is discussed in Section IV. The methodology is applied to case studies based on the Great Britain (GB) gas and electrical transmission networks, which are presented in Section V. Conclusions are given in Section VI. Finally, Appendix A discusses the evaluation of gas network supply flows, Appendix B describes the use of the finite-difference scheme in modelling the transient gas flow equations, and Appendix $\mathrm{C}$ gives a proof to a monotonicity property of steady-state gas flows used in the flexibility analysis.

\section{INTEGRATED GAS AND ELECTRICAL NETWORK MODELLING}

\section{A. Overall multi-stage methodology for integrated flexibility assessment}

At a given time $t_{0}$, the gas network flexibility is determined and the electrical constraints evaluated using the multistage model outlined in Fig. 1. This procedure begins with an assessment of the forecasted gas demand for electrical generation (gas and electrical networks are typically coupled at gas-fueled power stations and CHP facilities). This is carried out using a DC OPF with consideration of the forecasted meteorological conditions for the variable generation. The resulting gas demand for electricity generation is then combined with the non-power gas demand (which is highly correlated with the heat demand requirements) to determine the total forecasted gas demand (Stage A of Fig. 1). This is combined, in Stage B, with the gas network's state to evaluate the integrated gas and electricity flexibility metric using the steady-state gas flow modelling as part of the analysis (Section III). The flexibility metric is then used to define inter-network ramping constraints on the second OPF that aims at evaluating the dispatch of the generators. After evaluation of the gas demands and supplies for a given time snapshot, a transient gas flow is used to assess the state of the gas network at the consequent time period. In the application of the methodology in the case studies, following the UK electricity market arrangements, time periods of 30 mins have been considered for each iteration of Fig. 1. However, the methodology is more general and may be applied to systems with alternative operational and market arrangements. 


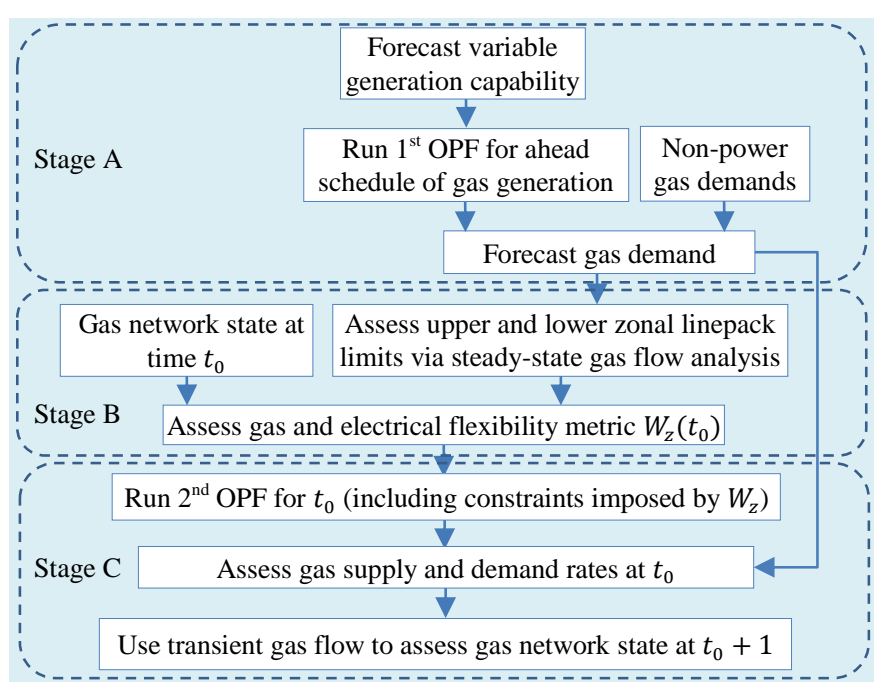

Fig. 1. Multi-stage integrated electricity and gas network flexibility modelling at each time step.

\section{B. "Stage A" OPF and assessment of forecasted gas demand}

As aforementioned, in Stage A the $1^{\text {st }}$ of two DC OPF analyses is conducted to assess the gas required for electrical generation for ahead scheduling of the gas system operation. More specifically, based on the forecasted generation capability $\overline{F P_{R G}(t)}$ of the renewable generators $R G$, for each time $t$ the OPF assesses the non-gas conventional generators $C G$ and gas turbines $G T$ forecasted real power outputs $\left(F P_{C G}\right.$ and $F P_{G T}$, respectively), as well as the actual output $F P_{R G}$ of the renewable generators. The $1^{\text {st }} \mathrm{OPF}$ is expressed as

$$
\text { minimize } \begin{aligned}
f_{1}(\boldsymbol{F P})=\sum_{C G} c_{C G} \cdot F P_{C G}( & t)+\sum_{G T} c_{G T} \cdot F P_{G T}(t) \\
& +\sum_{R G} c_{R G} \cdot F P_{R G}(t)
\end{aligned}
$$

subject to

$$
\boldsymbol{F H}(t)=\mathbf{X}^{-1} \mathbf{A}^{\mathrm{T}} \mathbf{B}^{-1} \boldsymbol{F U}(t)
$$

$$
\begin{aligned}
& F U_{b}(t)=\sum_{C G \in \mathrm{CGENS}_{b}} F P_{C G}(t)+\sum_{G T \in \mathrm{GGENS}_{b}} F P_{G T}(t) \\
& +\sum_{R G \in \mathrm{RGENS}_{b}} F P_{R G}(t)-E D_{b}(t) \\
& \left|F H_{l}(t)\right| \leq \overline{H_{l}} \\
& \underline{P_{C G}} \leq F P_{C G}(t) \leq \overline{P_{C G}} \quad \text { if } \quad f s_{C G}(t)=1 \\
& F P_{C G}(t)=0 \text { if } \quad f s_{C G}(t)=0 \\
& P_{G T} \leq F P_{G T}(t) \leq \overline{P_{G T}} \quad \text { if } \quad f s_{G T}(t)=1 \\
& F P_{G T}(t)=0 \text { if } f s_{G T}(t)=0 \\
& 0 \leq F P_{R G}(t) \leq \overline{F P_{R G}(t)} \\
& \sum_{C G} F R_{C G}(t)+\sum_{G T} F R_{G T}(t) \geq F S R(t) \\
& F R_{C G}(t)=f s_{C G}(t) \cdot \min \left\{\overline{R_{C G}}, \overline{P_{C G}}-F P_{C G}(t)\right\} \\
& F R_{G T}(t)=f s_{G T}(t) \cdot \min \left\{\overline{R_{G T}}, \overline{P_{G T}}-F P_{G T}(t)\right\}
\end{aligned}
$$

In (1): $\boldsymbol{F P}$ is the vector of forecasted real power outputs indexed by the generators; each non-gas conventional generator is modelled with a fixed marginal cost $c_{C G}$ while each of the gas turbines has cost equal to $c_{G T}=c_{g} / \eta_{G T}$, with $\eta_{G T}$ being the generator's efficiency and $c_{g}$ the cost of natural gas; and the renewable generators $R G$ are modelled with a marginal cost $c_{R G}$ equal to zero. The vector $F H$ of transmission line power flows are described by (2), where $\mathbf{B}$ is the admittance matrix, $\mathbf{A}$ the line-bus incidence matrix, $\mathbf{X}$ the diagonal matrix with the line impedances as entries, and $\boldsymbol{F} \boldsymbol{U}$ is the vector of net power injection $F U_{b}$ at each network bus $b$. Relation (3) describes this net power injection $U_{b}$, where $E D_{b}$ is the electrical demand and $\mathrm{CGENS}_{b}$ (resp. GGENS ${ }_{b}$, $\mathrm{RGENS}_{b}$ ) is the set of conventional non-gas (resp. gas, renewable) generators at bus $b$. Each transmission line $l$ has the magnitude of its real power flow $F H_{l}$ limited by $\overline{H_{l}}$ in (4). All conventional generators (gas and non-gas) are modelled with consideration of their installed capacity $\left(\overline{P_{C G}}\right.$ and $\left.\overline{P_{G T}}\right)$ and Minimum Stable Generation (MSG) levels $\left(\underline{P_{C G}}\right.$ and $\left.\underline{P_{G T}}\right)$ in (5) and (6), where $f s_{C G}(t)$ and $f s_{G T}(t)$ denote whether the generators are online or offline at $t$. Relation (7) bounds the actual renewable generation output with its maximum possible production at time $t$. Finally, each generator $C G$ and $G T$ contributes a certain amount of reserve $F R_{C G}$ and $F R_{G T}$ to fulfil the system reserve requirements $F S R$ in (8), with reserve provision from each generator limited by their 30 minute ramp rates $\overline{R_{C G}}$ and $\overline{R_{G T}}$ and the available generation headroom at time $t$, as from (9)-(10) [14].

If the results of the OPF indicate that the gas generator $G T$ has forecasted real power output $F P_{G T}(t)$ at time $t$, then the volumetric gas demand for this generator is given by $F_{G T}(t) /$ $\left(H H V \cdot \eta_{G T}\right)$, where $H H V$ is the higher heating value of the gas which describes its energy content per unit volume (in $\mathrm{MJ} / \mathrm{m}^{3}$ ). This first OPF forecasts the total gas demand for the gas generators

$$
F D_{P}(t)=\sum_{G T} F P_{G T}(t) /\left(H H V \cdot \eta_{G T}\right)
$$

which, when combined with the predicted non-power gas demand $F D_{N}(t)$, determines the overall forecasted gas demand

$$
F D(t)=F D_{P}(t)+F D_{N}(t)
$$

This forecasted demand is used for the assessment of the upper and lower linepack limits and of the network supply flows and gas network operation.

\section{Steady-state gas flow analysis}

The modelling of the time-ahead scheduling of the gas network requires the evaluation of the system with a steadystate gas flow analysis. In the formulation each pipe $\pi$ has gas flow rate $Q_{\pi}\left[\mathrm{m}^{3} / \mathrm{s}\right]$ which is related to its start and end pressures, $p_{\pi, 1}$ and $p_{\pi, 2}[\mathrm{~Pa}]$ by

$$
\operatorname{sign}\left(Q_{\pi}\right)\left|Q_{\pi}\right|^{\alpha}=K_{\pi}\left(p_{\pi, 1}^{2}-p_{\pi, 2}^{2}\right)
$$

where $\alpha>1$ is a constant which depends on the flow model used for the steady-state analysis and $K_{\pi}$ is a constant which depends on the gas flow model and pipe and gas characteristics. Within a gas network, compressor stations are usually used to maintain pressures caused by pressure drops. To each compressor station $C P$ is associated an inlet node $C P_{\text {in }}$ and outlet node $C P_{\text {out }}$ with corresponding pressures $p_{C P_{\text {in }}}, p_{C P_{\text {out }}}$ and a flow rate $Q_{C P}$. For a given gas network node $n$, if $\Pi_{n}$ are the pipes adjacent to $n$, and if there is at $n$ 
the outlet to compressor station $C P_{1}$ or inlet to compressor station $C P_{2}$, then the conservation of mass at $n$ is described by

$$
\sum_{\pi \in \Pi_{n}} Q_{\pi}+Q_{C P_{1}}-Q_{C P_{2}}=D_{n}-S_{n}
$$

where $D_{n}$ and $S_{n}$ are the demand and supply flows at node $n$. It is assumed that each compressor station $C P$ is modelled by a fixed compressor ratio $\beta_{C P}$, i.e.,

$$
p_{C P_{\text {out }}}=\beta_{C P} \cdot p_{C P_{\text {in }}}
$$

Finally, a slack pressure node $n_{s}$ is introduced into the modelling and associated with a fixed pressure

$$
p_{n_{s}}=p_{n_{s}}^{*}
$$

Resolving (13), (14), (15) and (16) for each node and pipe in the network defines its pressures and flows. Following [13] [15], these equations are solved using the Newton node method. The explicit and detailed application of the steadystate gas flow analysis in assessing the upper and lower linepack limits and as a step to determine the flexibility metric (Stage B of Fig. 1) is presented in Section III.

\section{$D$. $2^{\text {nd }} O P F$ with constraints from the gas network}

The integrated flexibility modelling defines limits on the ability of gas turbines to change their output based on the availability of gas in a given gas network zone $z$. As will be presented in Section III.C, the quantity $\overline{W_{z}\left(t_{0}\right)}$ imposes constraints on the ability for the generators to increase their output over a time period $T$. In Stage C of Fig. 1 , a $2^{\text {nd }} \mathrm{OPF}$ is used to assess the actual dispatch of the gas generators at $t_{0}$, including these inter-network ramping constraints while continuing to satisfy the other system and reserve requirements. This $2^{\text {nd }} \mathrm{OPF}$ is described by

$$
\begin{aligned}
& \text { minimize } f_{2}(\boldsymbol{P})=\sum_{C G} c_{C G} \cdot P_{C G}\left(t_{0}\right)+\sum_{G T} c_{G T} \cdot P_{G T}\left(t_{0}\right) \\
& +\sum_{R G} c_{R G} \cdot P_{R G}\left(t_{0}\right) \\
& \text { subject to } \quad \boldsymbol{H}\left(t_{0}\right)=\mathrm{X}^{-1} \mathrm{~A}^{\mathrm{T}} \mathrm{B}^{-1} \boldsymbol{U}\left(t_{0}\right) \\
& U_{b}\left(t_{0}\right)=\sum_{C G \in \mathrm{CGENS}_{b}} P_{C G}\left(t_{0}\right)+\sum_{G T \in \mathrm{GGENS}_{b}} P_{G T}\left(t_{0}\right) \\
& +\sum_{R G \in \mathrm{RGENS}_{b}} P_{R G}\left(t_{0}\right)-E D_{b}\left(t_{0}\right) \\
& \left|H_{l}\left(t_{0}\right)\right| \leq \overline{H_{l}} \\
& \underline{P_{C G}} \leq P_{C G}\left(t_{0}\right) \leq \overline{P_{C G}} \quad \text { if } \quad s_{C G}\left(t_{0}\right)=1 \\
& P_{C G}\left(t_{0}\right)=0 \text { if } \quad s_{C G}\left(t_{0}\right)=0 \\
& \underline{P_{G T}} \leq P_{G T}\left(t_{0}\right) \leq \overline{P_{G T}} \quad \text { if } \quad s_{G T}\left(t_{0}\right)=1 \\
& P_{G T}\left(t_{0}\right)=0 \text { if } s_{G T}\left(t_{0}\right)=0 \\
& 0 \leq P_{R G}\left(t_{0}\right) \leq \overline{P_{R G}\left(t_{0}\right)} \\
& \sum_{C G} R_{C G}\left(t_{0}\right)+\sum_{G T} R_{G T}\left(t_{0}\right) \geq S R\left(t_{0}\right) \\
& R_{C G}\left(t_{0}\right)=s_{C G}\left(t_{0}\right) \cdot \min \left\{\overline{R_{C G}}, \overline{P_{C G}}-P_{C G}\left(t_{0}\right)\right\} \\
& R_{G T}\left(t_{0}\right)=s_{G T}\left(t_{0}\right) \cdot \min \left\{\overline{R_{G T}}, \overline{P_{G T}}-P_{G T}\left(t_{0}\right)\right\} \\
& \sum_{G T \in G T_{z}}\left[P_{G T}\left(t_{0}\right)+R_{G T}\left(t_{0}\right)-F P_{G T}\left(t_{0}\right)\right] \cdot \Delta T_{E}
\end{aligned}
$$

$$
+\sum_{\substack{G T \in G T_{z} \\ t=t_{0}+\Delta T_{E}, t_{0}+2 \Delta T_{E} \ldots, t_{0}+T}} F R_{G T}(t) \cdot \Delta T_{E} \leq \overline{W_{z}\left(t_{0}\right)}
$$

where $P_{C G}, P_{G T}, P_{R G}$ are the real power outputs of the non-gas fueled generators, the gas fueled generators and the renewable generators, respectively, and $\boldsymbol{P}$ is the vector of real power outputs indexed by the generators. The maximum output $\overline{P_{R G}\left(t_{0}\right)}$ of each renewable generator is now limited by the prevailing meteorological conditions. The vector $\boldsymbol{U}$ of net power injections $U_{b}$ and $\boldsymbol{H}$ of transmission line real power flow $H_{l}$ are described by (18) and (19) with the latter continuing to satisfy the line constraints (20). The variables $s_{C G}$ and $s_{G T}$ describe whether $C G$ and $G T$ are online at $t_{0}$. The fact that the reserve $R_{C G}$ and $R_{G T}$ provided by the non-gas and gas conventional generators satisfies the system reserve requirements $S R\left(t_{0}\right)$ is modelled by (24)-(26). Finally, as key novelty and contribution, relation (27) imposes limits on the electrical network due to bounds on the flexibility of the gas network. More specifically, let us consider the flexibility modelling discussed in detail in Section III.C, and in particular the constraint (52) (reported here as (28) for clarity):

$$
\int_{t_{0}}^{t_{0}+T}\left[P_{G T_{z}}(t)+R_{G T_{z}}(t)-F P_{G T_{z}}(t)\right] d t \leq \overline{W_{z}\left(t_{0}\right)}
$$

where, for each zone $z, P_{G T_{z}}$ (resp. $F P_{G T_{z}}, R_{G T_{z}}$ ) is the power output (resp. forecast power output, reserve contribution) of the set of gas turbines in the zone. Then, upon the introduction of the time resolution $\Delta T_{E}$ (e.g., 30 mins) of the OPF simulation, the left-hand side of (28) can be approximated by

$$
\sum_{t=t_{0}, t_{0}+\Delta T_{E}, \ldots, t_{0}+T}\left[P_{G T_{z}}(t)-F P_{G T_{z}}(t)+R_{G T_{z}}(t)\right] \cdot \Delta T_{E}
$$

It is assumed that, at $t_{0}$, the OPF will assess the generator dispatch to fulfill the demands at $t_{0}$ while being able to satisfy the forecasted power system requirements determined by the forecast OPF. Hence, for $t>t_{0}, P_{G T_{z}}(t)$ is set to $F P_{G T_{z}}(t)$ and $R_{G T_{z}}(t)$ is set to $F R_{G T_{z}}(t)$. Therefore, the restrictions that the integrated gas and electrical network flexibility imposes on the dispatch of generators is expressed by (27), where the summations are over the collection of gas turbines in the zone $G T_{z}$.

\section{E. Assessment of actual gas requirements and gas network} state by transient flow analysis

The results of the $2^{\text {nd }}$ OPF now define the gas demand for generation for the next gas network transient analysis. Each gas generator $G T$ is associated with a gas node $n$ so that the gas demand for electrical generation at the node is given by

$$
D_{n, P}(t)=\sum_{\begin{array}{c}
\text { Gas turbine } G T \\
\text { at node } n
\end{array}} P_{G T}(t) /\left(H H V \cdot \eta_{G T}\right)
$$

The resulting gas demand at node $n$ is then given by

$$
D_{n}(t)=D_{n, P}(t)+D_{n, N}(t)
$$

where $D_{n, N}(t)$ is the non-power demand at $n$. These demands are then used in a transient gas flow analysis to model the gas network's state at a given time. This transient analysis is required for two reasons. Firstly, successful intraday 
modelling of gas networks and assessment of its state at a given time within the day is only possible with a transient gas flow which can account for its dynamic nature. Secondly, the network operation is based on the forecasted gas demand. As such, forecast errors in the demand may lead to gas network states with reduced flexibility. Therefore, in order to successfully assess this characteristic, it is necessary to consider the supply-demand flow imbalance via a transient gas flow analysis.

Upon unforecasted changes to the gas demand and consequent utilization of linepack, the gas network may be able to respond through, for example, a change in supply flows (e.g., from gas terminals). The operational practice of gas network shippers in changing supply flows, as well as its impact on the network's linepack, can vary widely for different networks. However, for networks such as the GB network, the shipper's response to forecast errors ${ }^{2}$ in demand can impact the network's flexibility. The change in system supply flows may be estimated through historical shipper actions and network characteristics and, in the case studies, are evaluated with consideration of the network's current state and forecast demand flows (see Appendix A for details).

For the transient gas flow, under the standard assumption of isothermal gas flow in a horizontal pipe, the pressures and flows in a section of pipe are described by three relations, namely, the equation of state (32) and the continuity and motion equations (33) and (34) [16]:

$$
\begin{gathered}
\frac{p}{\rho}=Z R_{\text {spec }} \Theta=e^{2} \\
\frac{e^{2}}{A} \cdot \frac{\partial M}{\partial x}+\frac{\partial p}{\partial t}=0 \\
\frac{1}{2} \frac{\partial p^{2}}{\partial x}\left(1-\frac{e^{2} M^{2}}{A^{2} p^{2}}\right)+\frac{2 e^{2} M|M|}{F^{2} d A^{2}} \\
+\frac{1}{A}\left(p \frac{\partial M}{\partial t}+\frac{e^{2}}{A} \frac{\partial M^{2}}{\partial x}\right)=0
\end{gathered}
$$

where $p$ is the pressure [Pa], $Z$ is the compressibility factor [dimensionless], $R_{\text {spec }}$ is the specific gas constant for natural gas $[\mathrm{J} / \mathrm{kg} \mathrm{K}], \Theta$ is the absolute temperature $[\mathrm{K}], \rho$ is the density $\left[\mathrm{kg} / \mathrm{m}^{3}\right], e$ is the speed of wave propogation in the gas $[\mathrm{m} / \mathrm{s}], M$ is the pipe flow rate $[\mathrm{kg} / \mathrm{s}], x$ is the distance along the pipeline $[\mathrm{m}], t$ is time $[\mathrm{s}], d$ is the pipe diameter $[\mathrm{m}], A$ is the pipe's cross-sectional area $\left[\mathrm{m}^{2}\right]$, and $F$ is the Fanning transmission factor [dimensionless]. Given a set of nodal supply and demand flows $S_{n}(t)$ and $D_{n}(t)$, the network flows, pressures, and hence linepack throughout the network are assessed with consideration of relations (32)-(34), the conservation of mass at each of the network nodes (14), and the compressor modelling (15), as discussed in Appendix B.

\footnotetext{
${ }^{2}$ For simplicity, and without loss of generality, only forecast errors in the gas generation demand due to wind generation (and the relevant need for reserve from flexible gas turbines), as assessed with the two OPFs, have been considered. However, the proposed framework could readily include other forecast errors too, for example for electrical demand and solar generation (by the OPFs), or for heat demand (whose impact on gas demand can be assessed within the forecasted non-power gas supply).
}

\section{INTEGRATED GAS AND ELECTRICITY FLEXIBILITY MODELLING}

This section develops the integrated gas and electricity flexibility modelling and introduces a relevant metric which defines limits on the ability of gas power stations to alter their consumption over a given timeframe. This metric may be used, for example, by gas network system operators in assessing whether a request by a gas power station to change their output can be accommodated by the gas network. The section begins with a general discussion of the "linepack" of a gas pipeline and introduces the concept of "linepack flexibility" which is developed here. There then follows the formulation of how these concepts have been extended from pipelines and applied to gas networks, also taking into account local requirements. Finally the integrated gas and electrical flexibility metric is presented in Section III.C.

As will be discussed, the methodology considers the forecasted gas network flows over the consequent 24 hours. To exemplify practical implementation, in the case studies which are based on the operational practice of the GB gas network, these are modelled considering hourly intervals.

\section{A. Linepack and pipeline flexibility}

The linepack of a pipe is defined as the quantity of gas it contains at a given time, usually measured with respect to the volume at normal temperature and pressure (NTP). If the pipe $\pi$ has volume $V$ and the start and end pressures are $p_{\pi, 1}$ and $p_{\pi, 2}[\mathrm{~Pa}]$ (with $p_{\pi, 1} \geq p_{\pi, 2}$ ), respectively, then if $p_{N T P}$ is the pressure at NTP, the linepack $L_{\pi}\left[\mathrm{m}^{3}\right]$ is given by [17]

$$
L_{\pi}=\frac{Z V}{p_{\mathrm{NTP}}} \frac{2}{3}\left(p_{\pi, 1}+p_{\pi, 2}-\frac{p_{\pi, 1} p_{\pi, 2}}{p_{\pi, 1}+p_{\pi, 2}}\right)
$$

Linepack plays a number of roles in gas network operation including maintaining minimum offtake pressures, maintaining flow characteristics, and allowing for variations in the supply and demand which may not be balanced instantaneously [17]. As such, not all the linepack is available to electrical generators. In particular, the linepack for transport requirements is defined as that necessary to support the expected gas flow operation while meeting minimum offtake pressures. It is assessed by considering the day's maximum flow conditions, which are dictated by the maximum supply or demand rate for the day. This has been modelled by considering the peak "non-power" gas demand (depending on the forecasted heat demand for the day) and the maximum gas demand for gas power plants ${ }^{3}$. The assessment of the maximum gas demand for power stations may consider, for example, the unit's capacity, its daily gas offtake flow limit, or other factors limiting its output within the day. In the modelling below and in the case studies it has been determined by considering their maximum power output. More specifically, if the gas turbine $G T$ has capacity $\overline{P_{G T}}$, then the maximum gas demand for the power station is given by

\footnotetext{
${ }^{3}$ As presented in [18], the pressure shock effects of sudden CCGT ramps is minor in comparison to pressure differences due to flow requirements, hence pressure variations due to ramp rates have been neglected and, conversely, restrictions on ramp rates have not been included as a limiting factor.
} 
$\overline{P_{G T}} /\left(H H V \cdot \eta_{G T}\right)$. Hence, if they are not constrained to operate at maximum capacity, this allows power stations to have the flexibility to change their power output profile over a given time period while consuming the same amount of gas. Hence, if the maximum power and non-power demand $\left[\mathrm{m}^{3} / \mathrm{s}\right]$ for the day is $\overline{D_{P}}$ and $\overline{D_{N}}$, respectively, then the flow $\overline{D_{P}}+\overline{D_{N}}$ at minimum and maximum pressure (Fig. 2) defines the minimum and maximum linepack for transport of the pipe.

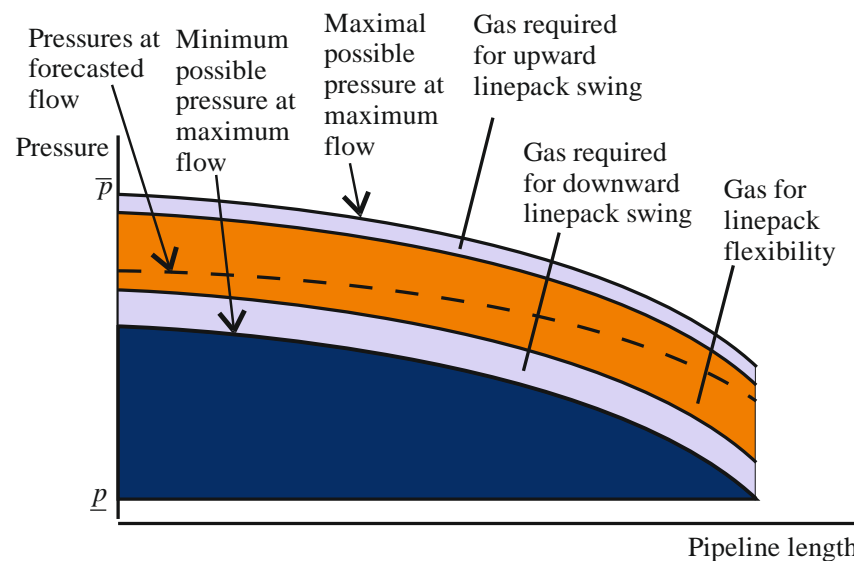

Fig. 2. Depiction of pressures in pipeline for alternative flows.

If $\underline{p}$ is the minimum operating pressure of the pipe $\pi$, then the end and start pressures with maximum flow are defined, respectively, by $p_{\pi, 2}=\underline{p}$ and, by using (13), $p_{\pi, 1}=$ $\sqrt{\underline{p}^{2}+\left(\overline{D_{P}}+\overline{D_{N}}\right)^{\alpha} / K_{\pi}}$. Using equation (35), the minimum linepack for transport $\underline{T R}$ is defined as:

$$
\begin{aligned}
\underline{T R}=\frac{Z V}{p_{\mathrm{NTP}}} \frac{2}{3}\left(\sqrt{\underline{p}^{2}+\left(\overline{D_{P}}+\overline{D_{N}}\right)^{\alpha} / K_{\pi}}+\underline{p}\right. \\
\left.-\frac{\underline{p} \sqrt{\underline{p}^{2}+\left(\overline{D_{P}}+\overline{D_{N}}\right)^{\alpha} / K_{\pi}}}{\sqrt{\underline{p}^{2}+\left(\overline{D_{P}}+\overline{D_{N}}\right)^{\alpha} / K_{\pi}}+\underline{p}}\right)
\end{aligned}
$$

Similarly, the maximum linepack for transport $\overline{T R}$ can be assessed by considering the maximum operating pressure $\bar{p}$, so that

$$
\begin{aligned}
\overline{T R}=\frac{Z V}{p_{\mathrm{NTP}}} \frac{2}{3}(\bar{p} & +\sqrt{\bar{p}^{2}-\left(\overline{D_{P}}+\overline{D_{N}}\right)^{\alpha} / K_{\pi}} \\
& \left.-\frac{\bar{p} \sqrt{\bar{p}^{2}-\left(\overline{D_{P}}+\overline{D_{N}}\right)^{\alpha} / K_{\pi}}}{\bar{p}+\sqrt{\bar{p}^{2}-\left(\overline{D_{P}}+\overline{D_{N}}\right)^{\alpha} / K_{\pi}}}\right)
\end{aligned}
$$

The quantity of gas in the pipe above $\underline{T R}$ is called the "linepack buffer" or "linepack reserve" [19]. In this work, the concept of linepack buffer has been extended in three aspects. Firstly, as well as the consideration of the lower limit on linepack $\underline{T R}$ as considered in [19], the introduction of the upper limit $\overline{T R}$ allows for the additional consideration as to the gas storage capacity of the pipe. Secondly, the inclusion of the temporal characteristics of the linepack allows for the evaluation of the constantly changing and dynamic nature of the buffer. Finally, we quantify how much of the linepack buffer is available for gas network flexibility.

The assessment as to how much of the linepack buffer is available for gas network flexibility first requires consideration of the linepack swing. "Linepack swing" is the cyclical change ${ }^{4}$ in the linepack which is a consequence of the demand-supply mismatch facilitated by the storage characteristics of the gas network, and is an important operational characteristic of gas networks. With technical and regulatory restrictions on the ability of gas suppliers to vary their flows on an hour-to-hour basis [20], daily linepack swing results from the supply flow being relatively flat compared to the demand profile. The change in linepack as a consequence of the daily linepack swing is exemplified in Fig 3. It is therefore necessary, so as to avoid pressure violations, that linepack is reserved for this intraday variation. The amount of gas required for this variation depends on the predicted demand and supply for the day. If $t_{0}$ is the start of the considered linepack swing cycle which ends at $t_{e}$ and the forecasted supply and demand are given by $F S(t)$ and $F D(t)$, $t \in\left(t_{0}, t_{e}\right)$, then the forecasted linepack swing $F S W(t)\left[\mathrm{m}^{3}\right]$ for this period is given by

$$
F S W(t)=\int_{t_{0}}^{t} F S(\tau)-F D(\tau) d \tau
$$

Then at each time $t$ we can consider an upper and lower linepack limit, $\bar{L}(t)$ and $\underline{L}(t)\left[\mathrm{m}^{3}\right]$ defined by

$$
\begin{aligned}
& \bar{L}(t)=\overline{T R}+F S W(t)-\max \left(\left\{F S W(\tau) \mid \tau \in\left(t_{0}, t_{e}\right)\right\}\right) \\
& \underline{L}(t)=\underline{T R}+F S W(t)-\min \left(\left\{F S W(\tau) \mid \tau \in\left(t_{0}, t_{e}\right)\right\}\right)
\end{aligned}
$$

and we call the interval $(\underline{L}(t), \bar{L}(t))$ the linepack flexibility at $t$.

A linepack flexibility utilization period has been incorporated into the modelling, which associates a timeframe over which gas in the network may be withdrawn or injected for flexibility. This period is dependent on the gas network as well as its ability to redispatch its operation. For example, larger gas networks, where changes to supply flows take longer to filtrate through the network before reaching demand, may consider a longer flexibility utilization period. Meanwhile, gas networks which actively manage their operation, for example through the use of compressor stations, may consider shorter time frames. If the linepack flexibility utilization period has length $T$, then we can describe the linepack flexibility (Fig. 3) as the space of all permissible temporal variations in linepack, expressed by

\footnotetext{
${ }^{4}$ Linepack swing may occur over a range of timescales, typically up to one week, according to different market practices and network configurations, interconnections and sizes. However, in the context of this work, following the natural demand cycle and common UK market arrangements, a reference period of one day (the most common average cycle length) is considered.
} 


$$
\left\{(L(t), t) \mid \underline{L}(t) \leq L(t) \leq \bar{L}(t), t \in\left(t_{0}, t_{0}+T\right)\right\}
$$

We can then assess the ability of the pipeline to change its supply/demand profile from that forecast in the interval $\left(t_{0}\right.$, $\left.t_{0}+T\right)$ by limiting the net change in linepack to $\bar{L}\left(t_{0}\right)-$ $L\left(t_{0}\right)$ and $\underline{L}\left(t_{0}\right)-L\left(t_{0}\right)$. The utilization of the linepack flexibility is exemplified in Fig. 3, where an increase in generation at $t_{0}$ causes a decrease in the pipe's linepack which remains within the limits defined by $\underline{L}(t)$ and $\bar{L}(t)$. After the withdrawal, the pipe is able to continue to operate satisfying flow and pressure requirements while at a reduced linepack.

Upon an increase in demand, gas may be replenished by increasing supply flows (from terminal or storage facilities) and the consequent impact may be assessed by updating the forecasted flows and re-evaluating the linepack flexibility for the consequent time period. Hence, the proposed methodology also allows for incorporation of the flexibility offered by other gas sources (and in particular storage) into the assessment. On the other hand, as mentioned above, the short-term (a few hours) response that the network may need in order to cope with fast changes in power plant dispatch can only be provided by the linepack flexibility discussed here.

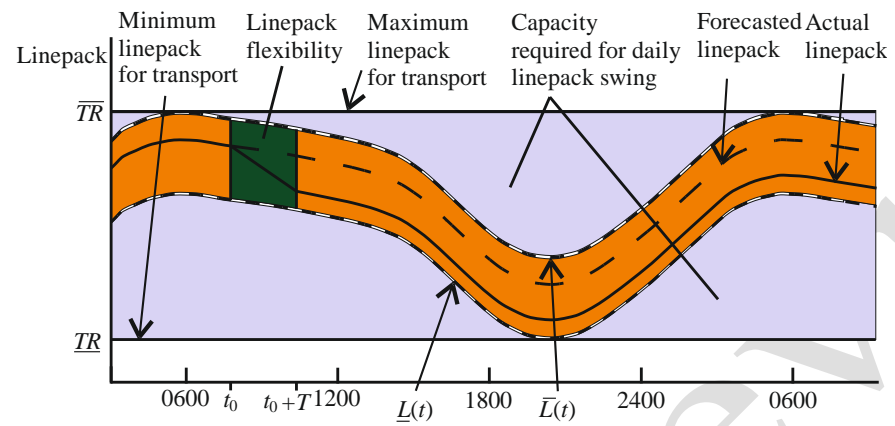

Fig. 3. Typical daily linepack variation, flexibility and example utilization.

\section{B. Gas network flexibility analysis and linepack zones}

When extending the concepts of linepack flexibility from a single pipe to a whole network, there are a number of further considerations. The linepack in the network will not be evenly distributed and some areas of the network may have greater limitations/potential in offering flexibility. As such, it can be beneficial to consider the partitioning of the network into linepack zones defined by collections of pipes; this is a practical viewpoint taken by the GB gas network system operator when operating the GB network based on rules and experience ${ }^{5}$. Each such zone $z$ will be assigned relevant indices so that $T R_{z}, \overline{T R_{z}}, \overline{L_{z}}(t)$, and $L_{z}(t)$ are analogous to $\overline{T R}, \underline{T R}, \bar{L}(t)$, and $\underline{L}(t)$ for a single pipe, respectively.

In order to formally estimate the linepack of each zone for a given system, a specific iterative procedure (see Fig. 4) has been developed. The procedure takes as an input a minimum/maximum system pressure or a predefined system

\footnotetext{
${ }^{5}$ Characteristics of the gas network which may be considered when defining the network's zones include the locality of terminals and storage facilities, compressor stations and the throughput capacity and volume of pipes, which all contribute to how gas flows are locally controlled and distribute throughout the network.
}

linepack and determines the steady-state gas flow for this system minimum/maximum pressure or linepack. Namely, as the network pressures of a steady-state gas flow are defined by the fixed pressure (slack) node [13], and, moreover, when the compressor stations are modelled with a fixed compression ratio, the pipe's pressures, and hence linepack, are monotonically increasing with respect to this slack pressure (see Appendix C), then iteratively evaluating the steady-state gas flow for varying slack pressure will lead to the desired steady-state gas flow corresponding to the minimum/maximum pressure or linepack. In particular, the bisection method is used to determine the slack pressure for each iteration while converging to the desired minimum/maximum pressure or linepack (Fig. 4). The resulting steady-state gas flow determines the pipe pressures and, using (35), their linepack. The linepack for each zone is determined by summing the linepack for each of the zone's pipes. This methodology is applied in the determination of the zones minimum (resp. maximum) transport linepack $T R_{z}$ (resp. $\overline{T R_{z}}$ ) for a day by considering the steady-state gas flow for the networks forecasted maximum power and non-power demands $\overline{D_{P}}$ and $\overline{D_{N}}$ along with the system's minimum and maximum pressures $p$ and $\bar{p}$.

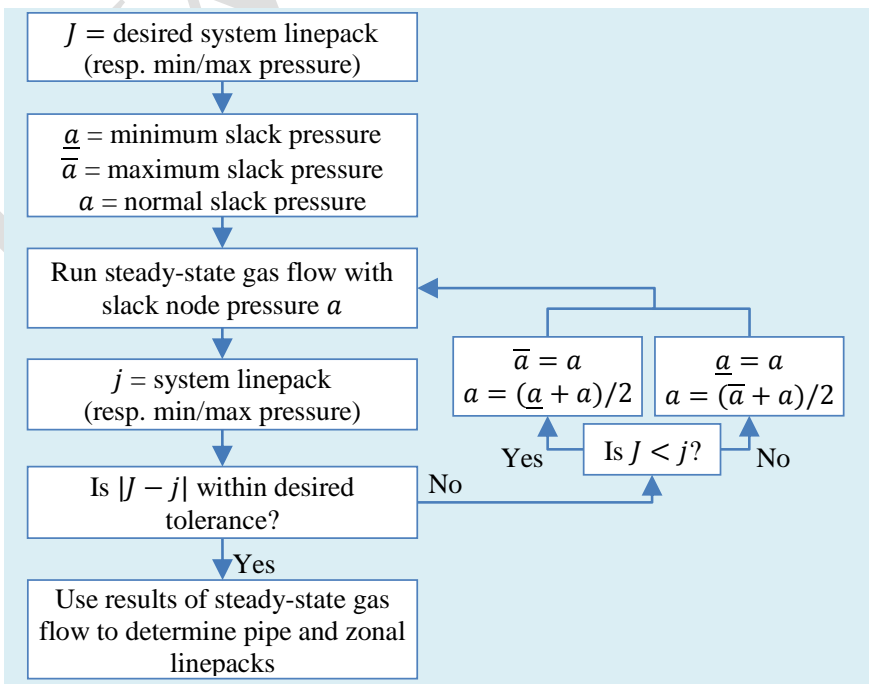

Fig. 4. Determination of zonal linepacks for a predefined system minimum/maximum pressure or linepack.

The methodology of Fig. 4 has also been used in the determination the upper and lower zonal linepack limits $\overline{L_{z}}(t)$ and $\underline{L_{Z}}(t)$, described below. For the system linepack $L\left(t_{0}\right)$ at $t_{0}$ and forecasted system linepack swing $F S W(t)$ as defined by (38) then the system forecasted linepack $F L(t)$ is given by

$$
F L(t)=L\left(t_{0}\right)+F S W(t)
$$

For each $t$, the system linepack $F L(t)$ is inputted into the procedure of Fig. 4 which returns the forecasted zonal linepacks $F L_{z}(t)$. The forecasted zonal linepack swings $\mathrm{FSW}_{z}(t)$ are then defined by

$$
F S W_{z}(t)=F L_{z}(t)-F L_{z}\left(t_{0}\right)
$$


Analogously to (39) and (40), the upper and lower zonal linepack limits are respectively defined by

$$
\begin{aligned}
& \overline{L_{z}}(t)=\overline{T R_{z}}+F S W_{z}(t)-\max \left(\left\{F S W_{z}(\tau) \mid \tau>t_{0}\right\}\right) \\
& \underline{L_{z}}(t)=\underline{T R_{z}}+F S W_{z}(t)-\min \left(\left\{F S W_{z}(\tau) \mid \tau>t_{0}\right\}\right)
\end{aligned}
$$

\section{Integrated gas and electrical network flexibility}

The extent of the upper and lower linepack flexibility depends on the networks state at $t_{0}$. If the linepack in zone $z$ is $L_{z}\left(t_{0}\right)$, then the quantity of gas $C_{z}\left(t_{0}\right)\left[\mathrm{m}^{3}\right]$ that may be withdrawn from the linepack has bounds given by

$$
\underline{C_{z}\left(t_{0}\right)} \leq C_{z}\left(t_{0}\right) \leq \overline{C_{z}\left(t_{0}\right)}
$$

where

$$
\begin{aligned}
& \overline{C_{z}\left(t_{0}\right)}=L_{z}\left(t_{0}\right)-\underline{L_{z}}\left(t_{0}\right) \\
& \underline{C_{z}\left(t_{0}\right)}=L_{z}\left(t_{0}\right)-\overline{\overline{L_{z}}}\left(t_{0}\right)
\end{aligned}
$$

The negative lower bound to $C_{z}\left(t_{0}\right)$ signifies limits on, for example, gas storage withdrawal while the upper bound limits the ability of the gas generators to increase their output. If $\eta_{z}$ is the efficiency ${ }^{6}$ of the gas generators in zone $z$, the increase in the total generation output $[\mathrm{MJ}]$ in the linepack flexibility utilization period is limited to

$$
\overline{E_{z}\left(t_{0}\right)}=H H V \cdot \overline{C_{z}\left(t_{0}\right)} \cdot \eta_{z}
$$

Based on the above, it is therefore possible to introduce an integrated gas and electrical flexibility metric describing the limitations imposed on gas generators modifying their output over a flexibility utilization period of duration $T$. More specifically, if the gas turbines $G T_{z}$ in zone $z$ have installed capacity $\overline{P_{G T_{z}}}[\mathrm{MW}]$ and, at $t_{0}$, have a forecasted power output $F P_{G T_{z}}(t)[\mathrm{MW}]$, then the gas and electrical flexibility metric $\overline{W_{z}\left(t_{0}\right)}[\mathrm{MJ}]$ is defined here as:

$$
\overline{W_{z}\left(t_{0}\right)}=\min \left(\int_{t_{0}}^{t_{0}+T}\left[\overline{P_{G T_{z}}}-F P_{G T_{z}}(t)\right] d t, \overline{E_{z}\left(t_{0}\right)}\right)
$$

In particular, if the power output of the generators is $P_{G T_{Z}}(t)$, then the metric imposes the constraint:

$$
\int_{t_{0}}^{t_{0}+T}\left[P_{G T_{z}}(t)-F P_{G T_{z}}(t)\right] d t \leq \overline{W_{z}\left(t_{0}\right)}
$$

The implementation of the restrictions imposed by $\overline{W_{z}\left(t_{0}\right)}$ may consider, as in (27) of the $2^{\text {nd }}$ OPF in Section II.D, the fulfillment of system reserve requirements. In this manner, the reserve contribution $R_{G T_{z}}$ of the generators in zone $z$ may also be included within the constraints, i.e.,

$$
\int_{t_{0}}^{t_{0}+T}\left[P_{G T_{z}}(t)+R_{G T_{z}}(t)-F P_{G T_{z}}(t)\right] d t \leq \overline{W_{z}\left(t_{0}\right)}
$$

This then also implies that not only generators' setpoints but also reserves may need to be rescheduled due to gas network flexibility constraints, so that power system flexibility is affected even more by these new inter-network constraints.

\footnotetext{
${ }^{6}$ For the sake of simplicity and without loss of generality, the efficiency is assumed to be the same for all gas generators in a linepack zone.
}

The gas and electrical network flexibility metric can also be applied at a system level to describe limits to the overall output of the system's gas turbines. More specifically, neglecting electrical system line constraints, a linear relationship between the gas zonal and system limitations may be assumed so that the integrated gas and electrical network flexibility metric for the whole system $\overline{W\left(t_{0}\right)}[\mathrm{MJ}]$ can be described by

$$
\overline{W\left(t_{0}\right)}=\sum_{z} \overline{W_{z}\left(t_{0}\right)}
$$

where the summation is over the linepack zones. The metric $\overline{W\left(t_{0}\right)}$ can be interpreted as a limit that the gas network imposes to the additional energy output of the gas turbines over the forthcoming hours and consequently a limit in their ability provide system balancing.

\section{EFFECTS OF LOW-CARBON HEATING SCENARIOS ON ELECTRICAL AND GAS TRANSMISSION NETWORK FLEXIBILITY}

In many cases (for instance in the GB system), the daily maximum non-power demand $\overline{D_{N}}$ is dominated by the gas demand for the heating sector. Changes to heat demand and technologies may therefore significantly affect the flexibility metric. In particular, electrification of the heating sector may lead to much greater variations in the net electrical demand (especially in the presence of renewables) and hence the gas turbine usage and ramping requirements. This will affect the magnitude of the daily linepack swing and hence also affect the flexibility metric. Finally, there will also be an increasing need to utilize the gas network's flexibility in accommodating any unpredicted ramps, again especially in the presence of large scale penetration of renewables.

In order to assess changes to the gas and electrical demand due to changes in heat generation technologies, previously developed models [21]-[23] that include statistics on building stock and lifestyle patterns have been combined to assess the heat demands at half-hourly intervals at regional levels. Each heat regional node is associated to an electrical bus and gas node so that the impact of changes to the heat technologies on the gas and electrical demands at each heat node are mapped against gas and electrical network node demands [13].

\section{CAse STUdies}

The integrated flexibility modelling developed has been applied to case studies based on the GB gas and electrical transmission networks and regional heat demands. To assess the impact of changes in the heating sector on the integrated gas and electrical network's flexibility, two cases based on possible heating scenarios for 2030 are considered:

- "Gas-based Heating", in which the heating sector remains dominated by gas-fueled technologies with an increased penetration of CHP;

- "Increased EHP", considers an increase in the proportion of heat produced through EHPs.

Below, details of the data used are presented. 


\section{A. Electrical network data}

The case studies are performed on a 29 busbar representation of the GB electrical transmission network (Fig. 5(a)). The generation portfolio considered is that put forward by National Grid in their Future Energy Scenarios under the Gone Green scenario at 2030 [24]. The capacity of each generator installed is presented in Table I as a fraction of the total installed capacity of $155 \mathrm{GW}$ along with the marginal generation cost, and, for the conventional generators, the MSG and $30 \mathrm{~min}$ ramp rates, each expressed as a fraction of the installed capacity. The cost of the gas-fueled generators depends on the gas price which is taken as $2.4 \mathrm{p} / \mathrm{kWh}$ as predicted in 2030 [24].

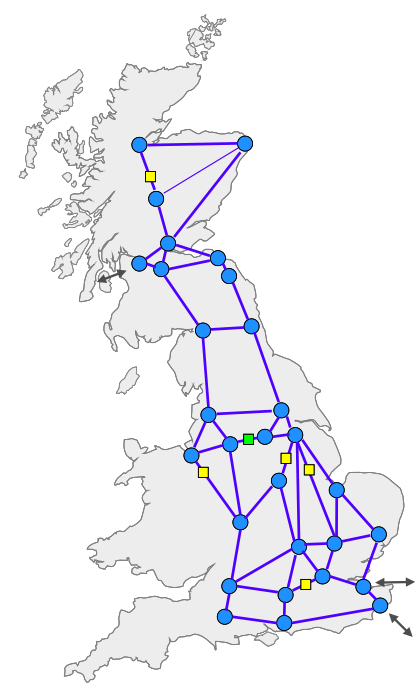

(a)

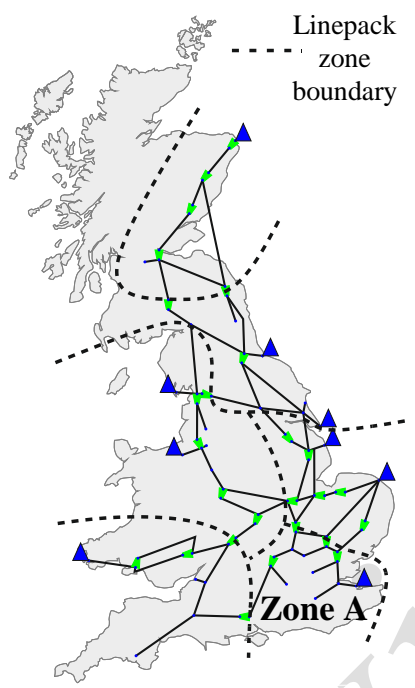

(b)
Fig. 5. 29 busbar electrical model (elaborated from [25]) (a) and 79 node gas model (with linepack zones) (b) of the GB transmission networks.

TABLE I

GENERATION TECHNOLOGY AND RESPECTIVE INSTALLED CAPACITY, COST, MSG AND 30-MIN RAMP RATES [13][24][26]

\begin{tabular}{|c|c|c|c|c|}
\hline Technology & $\begin{array}{l}\text { Installed } \\
\text { capacity }\end{array}$ & $\begin{array}{c}\text { Cost } \\
\left(\mathfrak{£} / \mathrm{MWh}_{\mathrm{e}}\right)\end{array}$ & MSG & $\begin{array}{c}30 \mathrm{~min} \\
\text { ramp rate }\end{array}$ \\
\hline CCGT & $22.3 \%$ & $\begin{array}{r}f(\text { gas } \\
\text { price })\end{array}$ & $50 \%$ & $25 \%$ \\
\hline CHP & $3.6 \%$ & Must run & - & - \\
\hline Coal (inc. CCS) & $7.1 \%$ & 46 & $50 \%$ & $25 \%$ \\
\hline Nuclear & $7.0 \%$ & Must run & - & - \\
\hline OCGT & $0.4 \%$ & $\begin{array}{r}f(\text { gas } \\
\text { price })\end{array}$ & $10 \%$ & $50 \%$ \\
\hline $\begin{array}{l}\text { Other } \\
\text { Renewables }\end{array}$ & $8.8 \%$ & 70 & - & - \\
\hline Marine & $4.8 \%$ & 0 & - & - \\
\hline Solar & $13.0 \%$ & 0 & - & - \\
\hline Wind & $33.0 \%$ & 0 & - & - \\
\hline
\end{tabular}

The solar and wind generation capability, i.e., the maximum possible generation without curtailment due to system constraints, has been assessed using historical meteorological conditions from 2004-05. For the solar profile, the levels of cloud cover at 10 locations throughout Britain have been considered. Each regional solar generation level at a given time is assessed considering the solar radiation and by using typical photovoltaics (PV) cell generation characteristics. Regarding wind generation, regional wind speeds from 39 locations in Britain have been combined with generation characteristics of a typical turbine to assess the wind generation output. The resulting generation capability for 12 18 December is illustrated in Fig. 6.

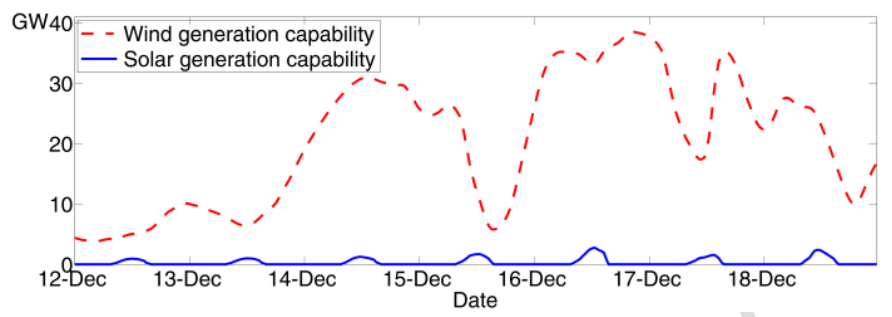

Fig. 6. Wind and solar generation capability in sample week.

\section{B. Gas network data}

The gas network modelling is conducted on a 79 node representation (Fig. 5(b)) of the GB National Transmission System (NTS). The NTS operates 24 hour balancing regimes which begin at 06:00 each day and it is assumed that, at this time, the system operator has planned the gas network operation for the day. In order to exemplify the proposed methodology, the flexibility modelling here follows this operational protocol. For the first OPF, a persistence approach is used so that the wind speeds at $t_{0}$ are used in the determination of the forecasted gas demand $F D(t)$ and the planning of the gas network operation for the consequent 24 hours. Changes to the gas demand throughout the day are balanced with a change in the terminal flows. Based on the operational characteristics of the GB terminals [20], there is a two hour delay between unforecasted changes to the gas demand and changes to the flow rate of the terminals (Appendix A). The minimum and maximum network pressures used in the determination of the flexibility metric are taken as 38 bar and 70 bar, respectively.

\section{Heat, electrical and gas demand data and analysis}

Historical temperatures and electrical demands from 201213 have been used to model the zonal heat requirements at half-hourly intervals for the 405 GB Local Authorities. For example, the system heat demand for the peak winter day is shown in Fig. 7.

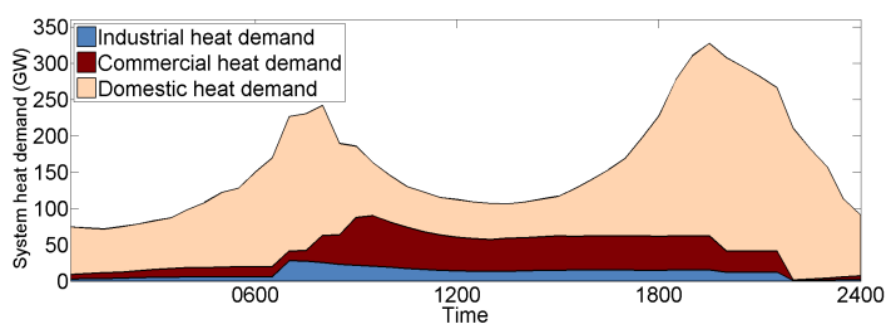

Fig. 7. Breakdown of system heat demand for peak winter day.

The gas and electrical requirements to fulfil this heat demand depends on the levels of heat producing technologies installed. For each of the two scenarios the level of penetration of the gas and electrical heating technologies, based on pathways put forward in [27], are described in Table II.

TABLE II

ANNUAL HEAT FULFILLED BY GAS AND ELECTRIC TECHNOLOGIES [13][27]

\begin{tabular}{l|ccc} 
& $\begin{array}{c}\text { Electric heat } \\
\text { pumps }\end{array}$ & $\begin{array}{c}\text { Gas } \\
\text { boiler }\end{array}$ & $\begin{array}{c}\text { Combined } \\
\text { heat-and-power }\end{array}$ \\
\hline Gas-based heating & $10 \%$ & $46 \%$ & $17 \%$ \\
Increased EHP & $39 \%$ & $50 \%$ & $0 \%$
\end{tabular}


The impact of alternative penetrations of heat producing technologies on the power sector is shown in Fig. 8 where, for each scenario, the generation output to meet the electrical demand is presented.

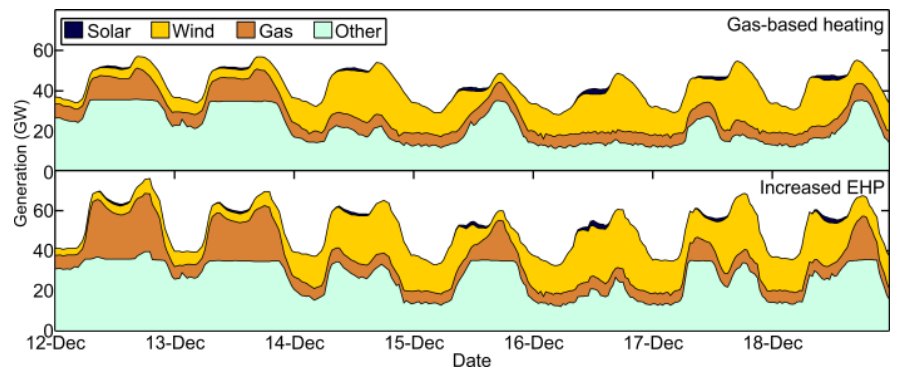

Fig. 8. Generation output to meet electrical demand for each scenario.

As an indication to the increased flexibility requirements of a power system with an increasingly electrified heating sector, Fig. 9 shows, the change over two hours in demand net of the renewable generation for the whole year, as returned by OPF modelling for the two scenarios considered. The results highlight a maximum 54\% (resp. 78\%) increase in the maximum upward (resp. downward) two hour ramps of the dispatchable generators. Furthermore, Fig. 10 shows, using historical meteorological data from 25 March 2004, how the combined effect of the evening reduction of solar generation and the increase in electricity demand for the heating sector in the Increased EHP scenario results in ramps of greater magnitude and length. In particular, the change in gas generation output between 16:00 and 19:00 is equal to $17 \mathrm{GW}$ with an average slope of $97 \mathrm{MW} / \mathrm{min}$ (Fig. 10(b)), against a ramp of $10 \mathrm{GW}$ and $57 \mathrm{MW} / \mathrm{min}$ average slope in the Gasbased scenario (Fig. 10(a)). This increase in the magnitude of the ramps follows from the utilization of gas generators as netload following units required to meet the greater variations in electrical demand with the electrification of heating and greater penetration of solar generation. Hence, countries considering such changes to the heating sector as a means of reducing carbon emissions should also consider potential issues such as additional integrated flexibility requirements.

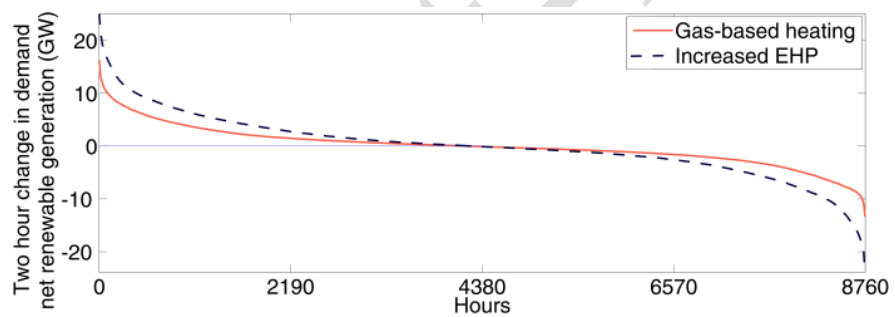

Fig. 9. Two hour change in demand net renewable generation for alternative heating scenarios.

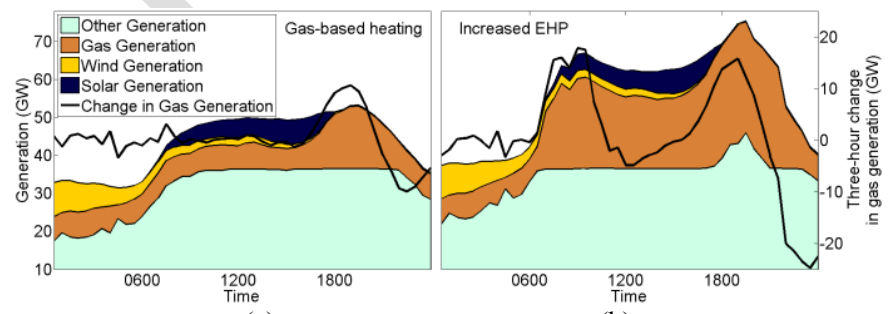

(a)

(b)

Fig. 10. Combined effect of heating technologies and high penetration of solar generation on ramps of gas generators ((a) Gas-based scenario; (b) Increased EHP scenario).

\section{Computational implementation of flexibility modelling}

The integrated flexibility modelling has been implemented in MATLAB and simulations conducted on a desktop computer with a $3.2 \mathrm{GHz}$ processor and 8GB RAM. To illustrate the computational requirements of the proposed integrated flexibility modelling framework, within each iteration of Fig. 1, the $1^{\text {st }}$ OPF takes on average 3 mins, the assessment of the integrated gas and electrical network flexibility approximately 5 mins, the $2^{\text {nd }} \mathrm{OPF}$ approximately 30 secs, and the transient gas flow analysis takes approximately 3 mins.

\section{E. Integrated gas and electrical flexibility analysis}

To analyze the impact of alternative heating scenarios on the combined networks' flexibility, the integrated gas and electrical flexibility metric discussed in Section III.C has been assessed for Zone A (Fig. 4(b)). Zone A has a large heat demand as well as $11 \mathrm{GW}$ of CCGT installed and so may be susceptible to the problem of reduced linepack. Hence, simulations were conducted with consideration of a flexibility utilization period of four hours. In Fig. 11, the integrated gas and electric flexibility metric for the week $12-18$ December is presented for each scenario. In particular, the picture highlights the constraints arising from the zonal integrated gas and electrical flexibility metric in (50), whereby the additional generation capability of CCGTs (described by their upward generation capacity $\left.\int_{t_{0}}^{t_{0}+T}\left[\overline{P_{G T_{Z}}}-F P_{G T_{Z}}(t)\right] d t\right)$ is compared to the limits imposed by the gas network's restriction to generation output $\overline{E_{z}\left(t_{0}\right)}$. In addition, the figure illustrates the effects of alternative heating technologies on the occasions when linepack flexibility imposes power system constraints. In particular, in the Gas-based scenario the number of hours in the week for which the gas generators in the zone would be incapable of fully utilizing their additional generation capacity due to restrictions in gas availability is equal to $90 \mathrm{~h}$, while in the Increased EHP scenario this number is equal to $42 \mathrm{~h}$.

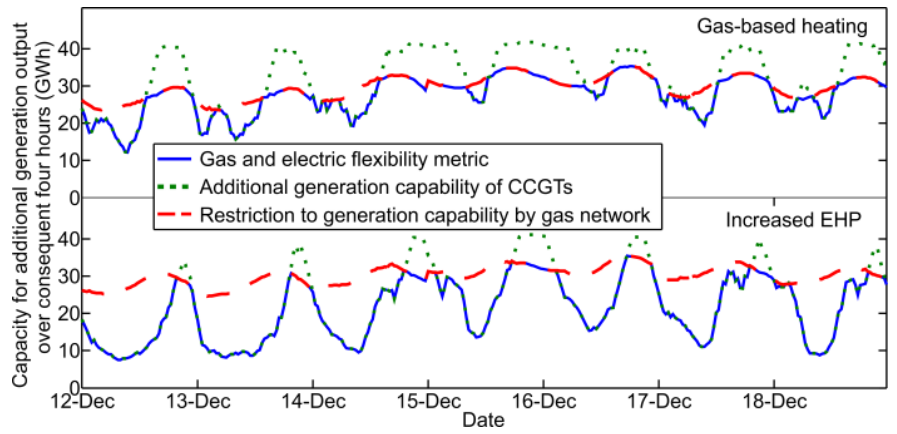

Fig. 11. Gas and electrical flexibility metric for alternative scenarios.

Considering the reserve allocation (described by $R_{G T}$ of (26)) then the introduction of gas-related flexibility constraints results in, for the Gas-based (resp. Increased EHP) scenario, a $27 \%$ (resp. 19\%) reduction in the reserve provided by the gas generators in zone A for the simulation week when compared to the analogous reserve allocation from the $1^{\text {st }} \mathrm{OPF}$. As mentioned earlier, this highlights the need to consider the gas network's capability at a given time when assessing power system's flexibility requirements and scheduling reserves, 
especially in the presence of different heating scenarios and a large amount of variable renewables.

\section{CONCLUSIONS}

In this work, a novel methodological framework, algorithmic implementation, and relevant metric have been presented for modelling and evaluation of integrated gas and electrical network flexibility, also taking into account changes in the heating sector, thus in the light of development of low-carbon multi-energy systems. This framework is based on integrated multi-stage electrical DC OPF and steady-state and transient gas network analysis modelling. The methodology allows for an evaluation of the ability of gas fueled generators to change their output throughout the day following net-load requirements, considering a regional evaluation of the available gas network flexibility in the form of zonal linepack and its usage by gas turbines. In this respect, the methodology specifically considers the constraints that gas network's local flexibility limits impose on the electrical network, by introducing new gas-related inter-network inter-temporal constraints in the "classical" OPF formulation. The proposed models have been applied in case studies on the GB gas and electrical transmission networks where the impacts of change in the heating sector on the integrated networks' flexibility and the power system's flexibility requirements and ability to provide reserve from local generators have been assessed.

The methodology and metric introduced may be incorporated into electrical system reliability studies to explore the impact of the gas network infrastructure on power system's generation and reserve scheduling and dispatch, as well as into integrated operational models to assess the combined reliability of low-carbon multi-energy systems with different renewables and heating scenarios.

Future work aims at exploring in more detail other types of flexibility interactions between electricity and gas networks, for instance considering power-to-gas technologies [28].

\section{APPENDIX A. ASSESSMENT OF GAS SUPPLY FLOWS}

The GB gas network operates with daily gas balancing in which gas shippers aim to balance their supply and demand flows over a 24-hour period and try to maintain flat supply flows. This appendix summarizes the assessment of the supply flows for the case studies. For a balancing period which begins at $B P_{s}$ and ends at $B P_{e}$, then, prior to the commencing of the period at $B P_{S}$ the forecasted supply flows for the 24 hours are presumed flat, i.e., the forecasted supplies at $B P_{S}$ are given by $F S_{B P_{S}}(t)=\int_{B P_{S}}^{B P_{e}} F D_{B P_{S}}(t) /\left(B P_{e}-B P_{s}\right) d t$, where $F D_{B P_{S}}(t)$ is the forecasted system demand at $B P_{S}$ for $t$. When there is a change in the forecasted demand, as discussed in Section III.E, there is a time period $T_{D}$ associated with the delay required for supply flows to respond to network changes (based on the response time of gas terminals [20], $T_{D}$ is taken as two hours in the case studies). Then, for $t_{0} \in\left(B P_{s}, B P_{e}-T_{D}\right)$, if the change in forecasted demand between iterations of Fig. 1 is $\Delta F D_{t_{0}}=\int_{t_{0}}^{B P_{e}} F D_{t_{0}}(t)-F D_{t_{0}-1}(t) d t$, then the supply flows are updated so that

$$
F S_{t_{0}}(t)= \begin{cases}F S_{t_{0}-1}(t)+\frac{\Delta F D_{t_{0}}}{B P_{e}-t_{0}-T_{D}} & \text { if } t-t_{0} \geq T_{D} \\ F S_{t_{0}-1}(t) & \text { if } t-t_{0}<T_{D}\end{cases}
$$

For the remaining time in the balancing period, i.e., when $t_{0} \in\left(B P_{e}-T_{D}, B P_{e}\right)$, then $\Delta F D_{t_{0}}$ is included in the demand for the consequent balancing period. The nodal supplies at $t_{0}$ are evaluated using historical shipper actions and are such that they sum to $F S_{t_{0}}\left(t_{0}\right)$. Similar approaches may also accommodate other market and balancing practices.

\section{APPENDIX B. TRANSIENT GAS FLOW MODEL}

As stated in Section II.E, the determination of the gas network state at a given time is assessed using transient gas flow analysis which is solved with a finite difference scheme. This appendix gives details on the construction of the scheme and the solution method. The analysis of the steady-state model is covered in Section II.C.

For a given section of pipe $s$ of length $\Delta x$ with, at $t_{0}$, start and end pressures $p_{s, 1}$ and $p_{s, 2}$ and start and end flow rates $Q_{s, 1}$ and $Q_{s, 2}$, then, at $t_{0}+\Delta t$, the start and end pressures $p_{s, 1}^{\prime}$ and $p_{s, 2}^{\prime}$ and flows $Q_{s, 1}^{\prime}$ and $Q_{s, 2}^{\prime}$ are described by approximating (33) and (34) by difference equations. The continuity equation (33) is approximated by

$$
\frac{\rho_{\mathrm{NTP}} R_{\text {spec }} \Theta}{A} \cdot \frac{\Delta t}{\Delta x}\left(Q_{s, 1}^{\prime}+Q_{s, 2}^{\prime}\right)+\frac{p_{s}^{\prime}}{Z_{s}}-\frac{p_{s}}{Z_{s}}=0
$$

while the motion equation (34) is approximated by

$$
\begin{aligned}
\frac{\left(p_{s, 1}^{\prime}\right)^{2}-\left(p_{s, 2}^{\prime}\right)^{2}}{2 \cdot \Delta x}+\frac{e^{2}}{F^{2} d A^{2}} \cdot \frac{\rho_{\mathrm{NTP}}^{2}\left(Q_{s, 1}^{\prime}+Q_{s, 2}^{\prime}\right)\left|Q_{s, 1}^{\prime}+Q_{s, 2}^{\prime}\right|}{2} \\
\quad+\frac{\rho_{\mathrm{NTP}} \cdot p_{s}}{A} \cdot \frac{Q_{s, 1}^{\prime}+Q_{s, 2}^{\prime}-Q_{s, 1}-Q_{s, 2 b}}{2 \cdot \Delta t}=0
\end{aligned}
$$

where $\rho_{\mathrm{NTP}}$ is the density of gas at NTP, and $p_{s}$ (resp. $Z_{S}$ ) is the pipe gas average pressure (resp. compressibility factor).

The system's flows and pressures at the next time step $t+\Delta t$ for all network pipe sections are then assessed by the solution of (14), (15), (55) and (56) for all pressures and flows using the Newton-Raphson method [16][28][29].

\section{APPENDIX C. MONOTONICITY OF PRESSURES AND LINEPACK WITH RESPECT TO SLACK PRESSURE IN THE GAS NETWORK}

In this appendix a proof is given for the fact, stated in Section III.B, that for given gas network demand and supply flows and with compressor stations modelled by fixed pressure ratio then the networks' pressures and linepack are monotonically increasing relative to the slack node pressure.

Let $n_{1}, n_{2}, \ldots$ be the network nodes, each node $n_{i}$ having an associated pressure $p_{n_{i}}$, and denote by $n_{s}$ the network's slack node. Considering a fixed set of nodal supply and demand flows and compressor pressure ratios, then for a set slack pressure $p_{n_{s}}=p_{n_{s}}^{*}$, suppose that there exists a network node $\left(n_{1}\right.$, say) such that $\left.\frac{d p_{n_{1}}}{d p_{n_{S}}}\right|_{p_{n_{S}}=p_{n_{S}}^{*}}<0$. Then there is a unique subset $\mathcal{N}$ of gas network nodes such that the following three conditions hold: $n_{1} \in \mathcal{N}$; for all $n_{i} \in \mathcal{N},\left.\frac{d p_{n_{i}}}{d p_{n_{S}}}\right|_{p_{n_{S}}=p_{n_{S}}^{*}}<0$; 
and, for all $n_{j} \notin \mathcal{N}$ such that there is a pipe connecting $n_{j}$ to some $n_{i} \in \mathcal{N}$ then $\left.\frac{d p_{n_{j}}}{d p_{n_{s}}}\right|_{p_{n_{S}}=p_{n_{S}}^{*}} \geq 0$. Note that as the compressors are modelled with a fixed pressure ratio then, for each compressor, both its inlet and outlet nodes will lie either within $\mathcal{N}$ or outside of $\mathcal{N}$. The relation defining the conservation of mass for the subset $\mathcal{N}$ then states that

$$
S_{\mathcal{N}}-D_{\mathcal{N}}+\sum_{\pi^{+}} Q_{\pi^{+}}-\sum_{\pi^{-}} Q_{\pi^{-}}=0
$$

where $S_{\mathcal{N}}$ (resp. $D_{\mathcal{N}}$ ) are the gas supplies (resp. demands) in $\mathcal{N}$ and $Q_{\pi^{+}}$are the flows for the pipes flowing into (including pipes of zero flow) $\mathcal{N}$ and $Q_{\pi^{-}}$the flows for the pipes flowing out of $\mathcal{N}$. It therefore follows that

$$
\left.\sum_{\pi^{+}} \frac{d Q_{\pi^{+}}}{d p_{n_{s}}}\right|_{p_{n_{s}=p_{n_{S}}^{*}}}-\left.\sum_{\pi^{-}} \frac{d Q_{\pi^{-}}}{d p_{n_{s}}}\right|_{p_{n_{S}}=p_{n_{S}}^{*}}=0
$$

Each pipe $\pi^{+}$flowing from $n_{j} \notin \mathcal{N}$ to $n_{i} \in \mathcal{N}$ has its flow $Q_{\pi^{+}}$related, by (13), to the pressures $p_{n_{j}}$ and $p_{n_{i}}$ so that

$$
\begin{gathered}
\left(Q_{\pi^{+}}\right)^{\alpha}=K_{\pi^{+}}\left(p_{n_{j}}^{2}-p_{n_{i}}^{2}\right) \\
\frac{d Q_{\pi^{+}}}{d p_{n_{s}}}=\frac{2 K_{\pi^{+}}}{\alpha\left(Q_{\pi^{+}}\right)^{\alpha-1}}\left(\frac{d p_{n_{j}}}{d p_{n_{s}}} p_{n_{j}}-\frac{d p_{n_{i}}}{d p_{n_{s}}} p_{n_{i}}\right)
\end{gathered}
$$

At the same time, the flows out of $\mathcal{N}$ satisfy

$$
\frac{d Q_{\pi^{-}}}{d p_{n_{s}}}=\frac{2 K_{\pi^{-}}}{\alpha\left(Q_{\pi^{-}}\right)^{\alpha-1}}\left(\frac{d p_{n_{i}}}{d p_{n_{s}}} p_{n_{i}}-\frac{d p_{n_{j}}}{d p_{n_{s}}} p_{n_{j}}\right)
$$

As $K_{\pi^{+}}, K_{\pi^{-}}, Q_{\pi^{+}}, Q_{\pi^{-}}, p_{n_{i}}, p_{n_{j}}>0$ and $\alpha>1$, then,

$$
\left.\frac{d Q_{\pi^{+}}}{d p_{n_{s}}}\right|_{p_{n_{S}}=p_{n_{S}}^{*}}>0 \quad \text { and }\left.\quad \frac{d Q_{\pi^{-}}}{d p_{n_{s}}}\right|_{p_{n_{S}}=p_{n_{S}}^{*}}<0
$$

which contradicts (58). Hence, for all $n_{i}$

$$
\left.\frac{d p_{n_{i}}}{d p_{n_{s}}}\right|_{p_{n_{S}=p_{n_{S}}^{*}}} \geq 0
$$

Therefore, for all $n_{i}$, the pressures at each node are monotonically increasing with respect to the slack pressure. It follows that the pressures within the pipes and, from (35), the linepack are also monotonically increasing.

\section{REFERENCES}

[1] Department of Energy and Climate Change, "The Future of Heating: Meeting the challenge," HM Government, London, March 2013.

[2] R. Baldick, "Flexibility and Availability: Can the Natural Gas Supply Support These Needs?," IEEE Power and Energy Magazine, vol. 12, no. 6, p. 100-104, Nov/Dec 2014.

[3] N. Keyaerts, E. Delarue, Y. Rombauts and W. D'haeseleer, "Impact of unpredictable renewables on gas-balancing in Europe," Applied Energy, vol. 119, p. 266-277, 2014.

[4] KEMA, "Integrating Gas and Electric Infrastructure in System Planning and Operations," in FERC Technical Conference, 2011.

[5] P. Mancarella, "MES (multi-energy systems): an overview of concepts and evaluation of models," Energy, vol. 65, no. 1, p. 1-17, Feb. 2014.

[6] E. Lannoye, D. Flynn and M. O'Malley, "Evaluation of Power System Flexibility," IEEE Transactions On Power Systems, vol. 27, no. 2, p. 922-931, 2012.

[7] J. Ma, V. Silva, R. Belhomme, D. Kirschen and L. F. Ochoa, "Evaluating and Planning Flexibility in Sustainable Power Systems," IEEE Transactions on Sustainable Energy, vol. 4, no. 1, p. 200-209, 2013.

[8] A. Ulbig and G. Andersson, "On Operational Flexibility in Power Systems," in IEEE PES General Meeting, San Diego, USA, 2012.
[9] S. An, Q. Li and T. W. Gedra, "Natural Gas and Electricity Optimal Power Flow," in Proceedings of IEEE PES Transmission and Distribution Conference, Dallas, USA, 2003.

[10] C. Liu, M. Shahidehpour, Y. Fu and Z. Li, "Security-Constrained Unit Commitment With Natural Gas Transmission Constraints," IEEE Transactions on Power Systems, vol. 24, no. 3, p. 1523-1536, 2009.

[11] M. Chaudry, N. Jenkins and G. Strbac, "Multi-time period combined gas and electricity network optimisation," Electric Power Systems Research, vol. 78, no. 7, p. 1265-1279, 2008.

[12] C. Liu, M. Shahidehpour and J. Wang, "Coordinated scheduling of electricity and natural gas infrastructures with a transient model for natural gas flow," Chaos, vol. 21, pp. 025102-1-025102-12, 2011.

[13] S. Clegg and P. Mancarella, "Integrated electrical and gas network modelling for assessment of different power-and-heat options," in Power Systems Computation Conference, Wroclaw, 2014.

[14] A. J. Wood and B. F. Wollenberg, Power Generation, Operation, and Control, Wiley-Interscience, 1996.

[15] A. J. Osiadacz, Simulation and analysis of gas networks, Gulf Publishing Co., 1987.

[16] V. L. Streeter and E. B. Wylie, "Natural Gas Pipeline Transients," Society of Petroleum Engineers Journal, vol. 10, no. 4, p. 357-364, 1970.

[17] N. Keyaerts, M. Hallack, J.-M. Glachant and W. D'haeseleer, "Gas market distorting effects of imbalanced gas balancing rules: Inefficient regulation of pipeline flexibility," En. Policy, vol. 39, p. 865-876, 2011.

[18] B. Rossi, J. Dalphin, L. Sinègre, A. Jacquiau and T. Renaudie, "Dealing with CCGT: An Explicit Dynamic Model," in Pipeline Simulation Interest Group Annual Meeting, 2011.

[19] DVGW, "Mindestanforderungen bezüglich Interoperabilität und Anschluss an Gasversorgungsnetze," Bonn, 2011.

[20] P. Wright, Gas Prices in the UK: Markets and Insecurity of Supply, Oxford University Press, 2006.

[21] N. Good, L. Zhang, A. Navarro-Espinosa and P. Mancarella, "High resolution modelling of multi-energy domestic demand profiles," Applied Energy, vol. 137, p. 193-210, 2015.

[22] A. Ahmed and P. Mancarella, "Strategic techno-economic assessment of heat network options for distributed energy systems in the UK," Energy, vol. 75, p. 182-193, 2014.

[23] E. Curioni, "UK domestic heat demand modeling," M.Sc. thesis, School of Electrical and Electronic Engineering, Un. of Manchester, UK, 2013.

[24] National Grid, "UK Future Energy Scenarios," 2013.

[25] K. R. W. Bell and A. N. D. Tleis, "Test system requirements for modelling future power systems," in IEEE Power and Energy Society General Meeting, 2012.

[26] V. L. F. P. Silva, "Value of flexibility in systems with large wind penetration," Ph.D. Thesis, University of London, July 2010.

[27] Deparment of Energy and Climate Change, "2050 Pathways Analysis," HM Government, 2010.

[28] S. Clegg and P. Mancarella, "Integrated Modeling and Assessment of the Operational Impact of Power-to-Gas (P2G) on Electrical and Gas Transmission Networks," IEEE Transactions on Sustainable Energy, vol. 6, no. 4, p. 1234-1244, 2015.

[29] I. Cameron, "Using an Excel-Based Model for Steady-State and Transient Simulation," in PSIG Annual Meeting, October 20-22, 1999.

\section{BIOGRAPHIES}

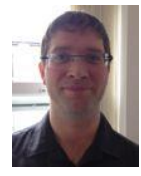

Stephen Clegg is at the University of Manchester where he is a research student in the Electrical Energy and Power Systems Group. His research includes the development of methods to study the interactions between the gas and electrical sectors and their applications to wider power systems contexts and integrated energy systems.

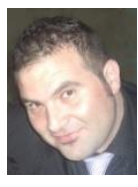

Pierluigi Mancarella (M'08, SM'14) received the Ph.D. degree in Electrical Engineering from Politecnico di Torino, Italy, in 2006. After working as a Research Associate at Imperial College London, UK, he is currently a Reader in Future Energy Networks at the University of Manchester, UK. His research interests include techno-economic and environmental analysis of multi-energy systems, business models for lowcarbon technologies, risk analysis of smart grid technologies, and infrastructure investment under uncertainty. $\mathrm{He}$ is an Associate Editor of the IEEE Systems Journal. 